\title{
The impact of the COVID-19 pandemic on women's mental health
}

\author{
Marcela Almeida $^{1,2}$ (1) $\cdot$ Angela D. Shrestha ${ }^{3} \cdot$ Danijela Stojanac $^{3} \cdot$ Laura J. Miller $^{4,5}$
}

Received: 9 June 2020 / Accepted: 4 November 2020 / Published online: 1 December 2020

(C) Springer-Verlag GmbH Austria, part of Springer Nature 2020

\begin{abstract}
The current worldwide outbreak of COVID-19 has changed the modus operandi of all segments of society. While some pandemic-related stressors affect nearly everyone, many especially affect women. Purpose: To review what is known about the pandemic's effect on women's mental health, what makes them more predisposed to vulnerabilities and adverse impacts, and strategies for preventing and treating these mental health consequences in the female population during specific stages across the lifespan. Methods: The authors performed a narrative review in combination with their observations from clinical experience in the field of women's mental health and reproductive psychiatry. Articles on women's mental health and COVID-19 up to May 30, 2020, were searched using the electronic PubMed and PsychInfo databases, as well as publications by major health entities (e.g., World Health Organization, Centers for Disease Control and Prevention, the United Nations) and press releases from prime communication outlets (e.g., National Public Radio). Results and conclusions: Women who are pregnant, postpartum, miscarrying, or experiencing intimate partner violence are at especially high risk for developing mental health problems during the pandemic. Proactive outreach to these groups of women and enhancement of social supports could lead to prevention, early detection, and prompt treatment. Social support is a key protective factor. Similarly, parenting may be substantially more stressful during a pandemic. Gender disparities may be accentuated, particularly for employed women or single parents, as women are disproportionately responsible for the bulk of domestic tasks, including childcare and eldercare.
\end{abstract}

Keywords COVID-19 $\cdot$ Reproductive psychiatry $\cdot$ women's mental health $\cdot$ Intimate partner violence $\cdot$ Pandemic

\section{Context, added value, and implications}

COVID-19 was first reported in Wuhan, China, in December 2019 , and rapidly spread to all parts of the globe, earning the title of a pandemic and changing the modus operandi of all segments of society. While some pandemic-related stressors influence nearly everyone, many especially affect women. In

This article is part of the Topical Collection on Women's mental health during the Covid-19 pandemic

Edited by: Anita Riecher-Rössler

Marcela Almeida

maralmeida@cha.harvard.edu

1 Department of Psychiatry, Harvard Medical School;Cambridge Health Alliance, Cambridge, MA 02139, USA

2 Department of Psychiatry, Cambridge Health Alliance, Cambridge, MA 02139, USA

3 University of Illinois at Chicago, Chicago, IL, USA

4 Loyola University Stritch School of Medicine, Maywood, IL, USA

5 Edward Hines Jr. VA Hospital, Hines, IL, USA this article, we review what is known about the pandemic's effect on women's mental health, and implications for preventing and treating these mental health consequences.

Although the global proportions of this pandemic are unprecedented, there are important lessons we can learn from prior major outbreaks, such as H1N1, Ebola, and Zika, where assessment and response delays resulted in negative outcomes (Hoffman and Silverberg 2018; O'Rourke et al. 2018). By doing a timely and comprehensive review and providing helpful recommendations, the authors hope to mitigate the devastating effects that it may cause in society, particularly in women.

\section{Methods}

This is a narrative review of published articles on women's mental health and COVID-19 up to May 30, 2020. The search used the electronic PubMed and PsychInfo databases, as well as publications by major health entities (e.g., World Health Organization, Centers for Disease Control and Prevention, the United Nations) and press releases from prime communication outlets (e.g., National Public Radio). 
Keywords and MeSH descriptor terms "novel coronavirus," "coronavirus," "SARS-CoV-2," "COVID-19," "pregnancy," "breastfeeding," "women's mental health," "intimate partner violence," "domestic violence," and "women's health" were used individually and in various combinations. An attempt to identify additional studies was made by reviewing the bibliographies from identified studies. No language restrictions were used.

Institutional review board approval and patient consent were not required. The authors' combined observations from clinical experience in the field of women's mental health were also included.

\section{Inclusion and exclusion criteria}

Inclusion and exclusion criteria were defined prior to initiating the systematic review. We included peer-reviewed articles on how women in particular are affected by SARS-CoV-2 and the current pandemic, including preconception, pregnancy, postpartum, and lactation, as well as the mental health impacts of quarantine and social isolation and their repercussions on the increased incidence of domestic violence. Opinion articles not substantiated by objective data were omitted.

\section{Gender influences on mental health effects of the COVID-19 pandemic}

Studies from China, where COVID-19 first surfaced, have found that female gender is significantly associated with higher self-reported levels of stress, anxiety, depression, and posttraumatic stress symptoms, and more severe overall psychological impact (Wang et al. 2020a, b; Liu et al. 2020a, b). Women have a higher prevalence of risk factors known to intensify during a pandemic, including chronic environmental strain (Street and Dardis 2018), preexisting depressive and anxiety disorders (Hao et al. 2020), and domestic violence (Campbell 2020).

In addition to influences associated with cultural gender roles, women also experience pandemic-related stressors specific to reproductive functioning and stages. Key influences are summarized below.

\section{Preconception and fertility issues}

Approximately $45 \%$ of pregnancies in the United States are unplanned or unintended (Finer and Zolna 2016, Ralph et al. 2020). This number may be elevated during the COVID-19 pandemic due to new barriers to access to contraception, from decreased office hours to attempts to avoid going to a healthcare facility to see a doctor or obtain a prescription, or cancellations of procedures for birth control, such as insertion of intrauterine devices, long-acting injections, or hormonal implants.
The unclear effects of SARS-CoV-2 on the developing fetus, limited therapeutic options, and reduced availability of resources have led many couples to face the difficult decision of whether to try to conceive or postpone pregnancy. For example, in a study in Italy, among survey participants who wanted a child prior to the COVID-19 pandemic, 37.3\% no longer did (Micelli et al. 2020). The most common reasons cited were economic concerns and worries about the effects of the pandemic on pregnancy. The postponement of childbearing may be especially stressful for women who have had to pause infertility treatments, or those nearing the end of their reproductive years with reduced access to assisted reproductive technology.

\section{Pregnancy}

The perinatal period (pregnancy and the first year postpartum) is a time of high vulnerability to mental health problems. Approximately one in seven perinatal women experience increased anxiety, depression, and distress. The risk is even higher among women experiencing a medically high-risk pregnancy (Fairbrother et al. 2017).

For many pregnant women, the COVID-19 pandemic brings increased fear and reduced sense of control due to the scarcity of definitive data on the effects of COVID-19 during pregnancy. During this period, key stressors include:

Worries about vulnerability, e.g.,

- Am I more vulnerable to COVID-19 infection or its complications while pregnant?

- Can COVID-19 be transmitted to my fetus?

- Does COVID-19 increase the risk of pregnancy complications?

Decisional stress, e.g.,

- Am I better off attending or skipping prenatal care visits?

- Should I avoid the hospital and deliver at home?

The fear of contagion and how it would affect the developing fetus adds another layer to the vast apprehension that the situation entails. Furthermore, pregnant women face additional unique challenges and experiences regarding perinatal care delivery during a pandemic:

- To reduce risk of COVID-19 exposure and transmission, many perinatal care visits have transitioned into a virtual mode.

- For in-person visits, several hospitals prohibit or limit, companions, or escorts (Hermann et al. 2020).

- Treatment teams have been reconfigured as healthcare workers are deployed to other areas or are unable to maintain their previous roles. 
- In many places, patients and providers need to follow a universal mask policy, which may have negative emotional associations and limit the patient-clinician rapport. Wearing a mask is especially anxiety-provoking for some women who have experienced prior trauma, e.g., women whose intimate partners have tried to suffocate them.

Studies to date indicate that pandemic-related stressors are substantially elevating perinatal mental health challenges. A study of 4124 pregnant women in China (Wu et al. 2020) compared scores on the Edinburgh Postnatal Depression Scale (EPDS), a well-validated screening and assessment tool, before and after January 20, 2020 (the day it was announced that SARS-CoV-2 could be transmitted from human to human). Compared to pregnant women before that announcement, pregnant women after that announcement had higher overall EPDS scores, greater likelihood of scoring above the cutoff for probable major depression, higher scores on the anxiety subscale, and greater likelihood of endorsing suicidal thoughts. Over time, EPDS scores correlated with the number of new COVID-19 cases and deaths announced each day. Similarly, a study of 1754 pregnant women in Canada (Berthelot et al. 2020) found that women recruited after the onset of the pandemic were almost twice as likely to report symptoms that met criteria for a diagnosis of depression, anxiety disorder, or substance use disorder. A different study of 1987 pregnant women in Canada (Lebel et al. 2020), using validated screening tools, found that $37.0 \%$ of participants endorsed elevated depressive symptoms (EPDS score 13 or above), $46.3 \%$ endorsed severely elevated anxiety, and $67.6 \%$ had clinically elevated pregnancyrelated anxiety. In that study, social isolation strongly correlated with the likelihood of clinically significant depression or anxiety. This is notable in that social support has been consistently shown to be a key protective factor, significantly reducing the risk of depressive and anxiety symptoms during pregnancy (Milgrom et al. 2019).

Fear of childbirth, very prevalent in some parts of the world (Koc et al. 2020), can be heightened when a woman is deprived of family support, is unfamiliar with the medical team, and worries that the baby will acquire the virus during pregnancy or delivery or that she will be separated from her newborn after birth. The unclear safety lingering ambiguity about the safety of breastfeeding with COVID-19 and the possibility that the newborn will be admitted to a neonatal intensive care unit represent additional sources of tension.

There are no uniform guidelines pertaining to the assessment and care of pregnant women during this pandemic, which leads to significant variability in management (Favre et al. 2020). To cite one example, in Bangalore, women in childbirth need to visit a police station and surrender their identification card to obtain a "childbirth pass" prior to heading to a hospital. The passes are valid for only 12 hours and many women are denied service (Decan Herald 2020a; 2020b).

Data about transplacental transmission of SARS-CoV-2 from infected pregnant women to their fetuses are ambiguous (Chen et al. 2020; Kimberlin and Stagno 2020; Ferrazzi et al. 2020; Egloff et al. 2020) and this ambiguity intensifies maternal stress. Some newborns whose mothers had COVID-19 have been found to have IgM antibodies to SARS-CoV-2, even when delivered by Cesarean section and immediately removed from their mothers, and even when mothers and all health personnel wore masks or other personal protective equipment (Dong et al. 2020; Zeng et al. 2020). Since IgM antibody molecules do not cross the placenta, it is posited that these represent an immune response mounted by the fetus due to in utero exposure. However, the IgM antibodies disappeared more quickly than expected, suggesting that these could have been false positives. In addition, some newborns have tested positive for SARS-CoV-2 despite immediate separation from mother at the time of birth, and some have had radiologic findings in the lungs, lymphocytopenia, and thrombocytopenia (Zaigham and Andersson 2020).

In terms of other complications when a pregnant woman has COVID-19, the most common adverse pregnancy outcome has been preterm birth, in 19-47\% of cases (Di Mascio et al. 2020; Mullins et al. 2020; Juan et al. 2020; Li et al. 2020). Much of this is iatrogenic. Other observed complications are premature rupture of membranes (19\% of cases), fetal distress (43\% of cases), and late perinatal loss (stillbirth or neonatal death, $7 \%$ of cases) (Di Mascio et al. 2020). Although initial data have not suggested an increased rate of miscarriage (Yan et al. 2020), there is at least one case of second-trimester miscarriage reported in a woman with COVID-19 that appears related to placental infection with SARS-CoV-2, supported by virological findings in the placenta (Baud et al. 2020). Contamination at the time of delivery, sampling, or laboratory error were unlikely, as all other swabs were negative for SARS-CoV-2. (American College of Obstetricians and Gynecologists 2020)

Compared to prepandemic placental tissue, placentas of women with COVID-19 have shown significantly more abnormal maternal (but not fetal) vascular perfusion, especially affecting maternal arterioles, and more intervillous thrombi (Shanes et al. 2020).

\section{Postpartum}

The postpartum period brings additional pandemic-related worries, such as:

- Is my newborn more vulnerable to COVID-19?

- Is it safe to hold my baby close?

- Is COVID-19 transmitted through breast milk? 
- If I have COVID-19, is it safe to store my breast milk and use it later?

- Is it safe to use a rented or shared breast pump? How do I disinfect the pump safety?

- What if I cannot find formula or diaper wipes due to stockpiling?

- How do I manage without my relatives coming to help?

- How can I support this baby now that I'm unemployed?

Social support is strongly protective against postpartum depression. The degree of social support significantly and inversely correlates with postpartum depressive symptom severity (Pao et al. 2019). Effective postpartum social support can include reliance on family, friends, or hired professional help to get some relief from added responsibilities while dealing with profound hormonal changes, sleep deprivation, and adjustments in the family dynamic and distribution of roles. With pandemicrelated stay-at-home or shelter-in-place orders, this is no longer an option for many mothers, who now find themselves juggling multiple roles with limited help (Brooks et al. 2020).

Many postpartum mothers and their partners are also dealing with added tasks such as childcare if there are other children in the home, since schools and childcare centers have closed in many places for an indefinite period, or the family has chosen to keep them at home due to fears of contamination. Single mothers are hit even harder. Elderly grandparents may be unable to assist, or even visit, due to heightened agerelated COVID risk.

Financial hardship is another significant factor, with unemployment rates at levels not seen since the Great Depression. In the United States, over 50 million people have filed claims for unemployment benefits, representing a third of the labor force (Department of Labor 2020). Many families, unable to afford rent, are being evicted and becoming homeless, and even shelters are being closed as they become infected.

With regards to lactation, to date, SARS-CoV-2 viral isolates have rarely been found in breast milk (Berveiller et al. 2020; Lackey et al. 2020). There are only a few case reports of women newly diagnosed with COVID-19 shortly after delivery who breastfed without masks, whose newborns tested positive for COVID-19 on day 1 and day 3 (Ferrazzi et al. 2020). Due to this ambiguity, there is no consensus about guidelines to address breastfeeding in the COVID-19 context. Some (Rasmussen et al. 2020) have recommended against breastfeeding in cases of confirmed infection. Most, however, support breastfeeding with precautions in place. For example, the American Academic of Pediatrics recommends that we "continue to promote and support breastfeeding in a family with suspected or confirmed COVID -19 since breastfeeding infants are less likely to have severe respiratory symptoms". They comment on the importance of the oxytocin released during breastfeeding to promote maternal wellness and stress relief and recommend that in cases where the mother is
COVID- 19 positive, infants with negative SARS-COV-2 molecular testing should be discharged to the care of a designated non-infected caregiver (American Academy of Pediatrics 2020). The Center for Disease Control (CDC) recommends that a mother wear a facemask and wash her hands with soap and water before each feeding. (Centers for Disease Control and Prevention 2020)

\section{Miscarriage}

Compared to women who maintain viable pregnancies, women who miscarry have higher rates of depression, anxiety, and posttraumatic stress symptoms (Farren et al. 2016). In nonpandemic conditions, women with non-viable pregnancies can opt for expectant care (natural miscarriage), medical treatment, or surgical treatment. During the pandemic, more may opt to miscarry at home due to wanting to reduce the risk of COVID-19 exposure in a medical setting. Compared to surgical treatment, natural miscarriage takes longer, is more likely to be incomplete and to require unplanned surgical intervention and/ or transfusions (Nanda et al. 2012). Women who would otherwise have opted for surgical intervention may now experience several days of bleeding and cramps, sometimes seeing fetal parts in the material they pass, and often experiencing this in more isolation, with less available social support. This suggests a higher risk for subsequent mental health problems.

\section{Parenting}

COVID-19 has redefined family life, maybe for generations to come. Schools were instantly closed in most countries as an attempt to contain the spread of the pandemic (https://en. unesco.org/covid19/educationresponse), affecting approximately 1.2 billion students or almost three-fourths of all the learners worldwide. A heavy load of parental stress came as a consequence, particularly for mothers, who typically carry the bulk of childcare and eldercare in most parts of the world. Many parents reported feeling more anxious, agitated, fearful, or depressed due to limited financial and social resources, unemployment, increased use of alcohol and other addictive substances, and a global sense of unpredictability and loss of control from the juggle between homeschooling and remote work all combined with limited outsourced help (https://www.parentingincontext. org/uploads/8/1/3/1/81318622/research_brief_stress_and parenting during the coronavirus_pandemic_final.pdf). This is substantially magnified in the case of single parents or parents with disabilities.

This sometimes translates as an increased risk of violence toward children. In addition to the caregivers' stress, children are facing their own struggles, having to adapt to a new routine, or lack thereof, while deprived of social and physical 
activities, so essential in their stage of life, and having increased exposure to screen time, irregular sleep patterns, and less balanced diets (Wang et al. 2020b).

The American Psychological Association recommends some strategies to mitigate these conflicts, such as establishing a structured routine, acknowledging one's feelings, relaxing screen time rules, practicing self-care (e.g., good sleep hygiene, healthy eating habits, meditation, yoga), and getting (virtual) professional help if necessary. (American Psychological Association 2020)

Reorganization of time and responsibilities is also important to prevent an exacerbation of gender inequities given how unbalanced the division of work tends to be, women spending significantly more time on housework and childcare and other unpaid work compared with men, and mothers under even greater disadvantage compared to childless people (Minello 2020).

It is possible, however, to find a silver lining amidst these very challenging times. While health emergencies and natural disasters have serious implications, including increased rates of child abuse and intimate partner violence, they may also present as opportunities to build strengths and positive behaviors, deepen interpersonal relationships, enhance interactions between parents and children, and develop new skills and abilities (Cluver et al. 2020). Many families have reported increased cohesion and partnership and a deeper understanding of which values are perceived as priorities for their family. Many mention that this experience has allowed them to return to previous hobbies or to develop new ones, and that spending more time with their immediate family has been incredibly positive.

\section{Intimate Partner Violence (IPV)}

As the world is told to stay at home in order to ensure safety, for many people home represents the least safe option. These are the victims of intimate partner violence, usually women, now with greater exposure to perpetrators of violence during a time of unprecedented psychological and economic stress, and with reduced access to safe havens. In some cases, violence may even develop in homes where it had not been an issue before.

According to the Centers for Disease Control and Prevention (2020), one in three women in the USA has experienced violence from an intimate partner. The World Health Organization calls domestic violence "the most widespread but among the least reported human rights abuses" (World Health Organization 2020). Violence in the home is linked to several negative outcomes, including increased use of substances, risky sexual behaviors, higher risk for chronic medical illnesses, depression, and posttraumatic stress disorder.

Rates of intimate partner violence have increased in the context of prior natural disasters. During and after Hurricane Harvey, for example, higher rates of domestic violence, including both intimate partner violence and child abuse, were observed (Schwartz et al. 2018). Contributory factors included reduced access to resources and social supports coupled with increased stress due to job loss and/or strained finances. Data so far indicate that a similar increase is happening during the COVID-19 pandemic, which compared to prior disasters includes especially prolonged shelter-in-place mandates. Increasing rates of domestic violence have been reported across the globe. There have been substantial documented increases in calls to (IPV) emergency support lines in China, Italy, and Spain during their lockdowns. Calls to Vancouver Battered Women's Support Services in Canada have tripled (Bradley et al. 2020). In France, there has been a 30\% increase in those calls, and in Brazil the number is even higher. In China, domestic violence is reported to have tripled during their shelter in-place mandate (National Public Radio 2020). Latin America, where femicide rates are among the highest in the world, has seen a dramatic rise in reports of domestic violence (Organisation for Economic Co-operation and Development 2020). It is noteworthy that these numbers are likely underrepresented, as many victims are deprived from any contact with the outside world or are fearful of retaliation. According to the United Nations, there has been a "horrifying surge" in domestic violence since the beginning of the pandemic (United Nations 2020). The organization urged that the prevention and redress of violence against women be a key part of national response plans for COVID-19 (United Nations 2020).

Due to isolation guidelines in effect in many places, victims of intimate partner violence are deprived of the option to stay with a friend or family member or go to a domestic violence shelter. Even filing a protective order can be a challenge. Many do not seek medical care in order to avoid the risk of COVID-19 exposure.

As current measures to limit the spread of COVID-19 trigger increases in intimate partner violence worldwide, physicians and frontline healthcare workers must be vigilant in recognizing signs of violence, feel comfortable asking about IPV, and be aware of local resources for referral (Campbell 2020). The World Psychiatric Association has issued a Position Statement on Intimate Partner Violence and Sexual Violence Against Women and developed a curriculum to support research, enable professionals, educate the public, and expand resources that address this theme (https://www. wpanet.org/position-statements; Stewart and Chandra 2017).

\section{Conclusions and clinical implications}

The COVID-19 pandemic may have exacerbated genderlinked mental health challenges. Available data about gender-specific effects suggest the following: 
- Women who are pregnant, postpartum, miscarrying, or experiencing intimate partner violence are at especially high risk for developing mental health problems during the pandemic. Proactive outreach to these groups of women could lead to prevention, early detection, and prompt intervention.

- Social support is a key protective factor. It can be enhanced via online contact-e.g., secure messaging with clinicians, telehealth visits, online support groups, online doula support.

- For pregnant women who may need to undergo labor and delivery without a loved one present, childbirth education can be adapted to include more stress management techniques, and can be delivered online.

- Parenting may be substantially more stressful during a pandemic. Offering specific parenting tips, such as those provided by UNICEF, could improve mental health for parents and children and reduce the risk of domestic violence. (UNICEF 2020)

- For women at risk of intimate partner violence, maintaining up-to-date information about available hotlines, shelters and family courts could be lifesaving.

- Gender disparities may be accentuated, particularly for employed women or single parents, as women are disproportionately responsible for the bulk of domestic tasks, including childcare and eldercare.

\section{Compliance with ethical standards}

Conflict of interest The authors declare that they have no conflicts of interest.

\section{References}

American Academy of Pediatrics. Breastfeeding guidance post hospital discharge for mothers or infants with suspected or confirmed SARSCo V-2 infection. https://services.aap.org/en/pages/2019-novelcoronavirus-covid-19-infections/breastfeeding-guidance-posthospital-discharge/. Accessed 18 Sept 2020

American College of Obstetricians and Gynecologists (2020). ACOG COVID-19 panel: what obstetrician-gynecologists need to know about the pandemic. https://www.acog.org/news/news-articles/ 2020/04/acog-covid-19-panel-what-obstetrician-gynecologistsneed-to-know-about-the-pandemic. Accessed Apr 2020

American Psychological Association (2020) How COVID-19 may increase domestic violence and child abuse. https://www.apa.org/ topics/covid-19/domestic-violence-child-abuse. Accessed Apr 2020

Baud D, Greub G, Favre G, Gengler C, Jaton K, Dubruc E, Pomar L (2020) Second-trimester miscarriage in a pregnant woman with SARS-CoV-2 infection. JAMA Published online April 30:21982200. https://doi.org/10.1001/jama.2020.7233

Berthelot N, Lemieux R, Garon-Bissonnette J, Drouin-Maziade C, Martel É, Maziade M (2020) Uptrend in distress and psychiatric symptomatology in pregnant women during the COVID-19 pandemic. Acta Obstet Gynecol Scand 99:848-855. https://doi.org/10.1111/aogs. 13925
Berveiller P, Guerby P, Garabedian C (2020) COVID19 and breastfeeding: not that simple. J Hum Lact 36(2):369-370. https:// doi.org/10.1177/0890334420917102

Bradley NL, DiPasquale AM, Dillabough K, Schneider PS (2020) Health care practitioners' responsibility to address intimate partner violence related to the COVID-19 pandemic. CMAJ. 192(22):E609-E610. https://doi.org/10.1503/cmaj.200634

Brooks SK, Webster RK, Smith LE et al (2020) The psychological impact of quarantine and how to reduce it: rapid review of the evidence. Lancet 395(10227):912-920

Centers for Disease Control and Prevention (2020) Preventing intimate partner violence https://www.cdc.gov/violenceprevention/ intimatepartnerviolence/fastfact.html. Accessed Oct 2020

Campbell AM (2020) An increasing risk of family violence during the Covid-19 pandemic: Strengthening community collaborations to save lives. Forensic Science International: Reports 2:100089. https:// doi.org/10.1016/j.fsir.2020.100089

Chen H, Guo J, Wang C et al (2020) Clinical characteristics and intrauterine vertical transmission potential of COVID-19 infection in nine pregnant women: a retrospective review of medical records [published correction appears in Lancet. 2020 Mar 28;395(10229): 1038] [published correction appears in Lancet. 2020 Mar 28;395(10229):1038]. Lancet. 395(10226):809-815. https://doi. org/10.1016/S0140-6736(20)30360-3)

Cluver L, Lachman JM, Sherr L et al (2020) Parenting in a time of COVID-19 [published correction appears in Lancet. 2020 Apr 11;395(10231):1194]. Lancet 395(10231):e64. https://doi.org/ 10.1016/S0140-6736(20)30736-4

Decan Herald (2020a) COVID-19: Cancer survivors, pregnant women hit hard. https://www.deccanherald.com/city/top-bengaluru-stories/ covid-19-cancer-survivors-pregnant-women-hit-hard-823114.html. Accessed Apr 2020

Decan Herald (2020b) COVID-19 lockdown: Bengaluru Police to issue 12-hour pass for medical emergency. https://www.deccanherald. $\mathrm{com} /$ city/top-bengaluru-stories/covid-19-lockdown-bengalurupolice-to-issue-12-hour-pass-for-medical-emergency-820309.html. Accessed Apr 2020

Department of Labor (2020) Coronavirus resources https://www.dol. gov/. Accessed 12 Nov 2020

Di Mascio D, Khalil A, Saccone G et al (2020) Outcome of coronavirus spectrum infections (SARS, MERS, COVID 1-19) during pregnancy: a systematic review and meta-analysis. Am J Obstet Gynecol MFM 25:100107. https://doi.org/10.1016/j.ajogmf.2020.100107

Dong L, Tian J, He S, Zhu C, Wang J, Liu C, Yang J (2020) Possible vertical transmission of SARS-CoV-2 from an infected mother to her newborn [published online ahead of print, 2020 Mar 26]. JAMA. 323(18):1846-1848. https://doi.org/10.1001/jama.2020.4621

Egloff C, Vauloup-Fellous C, Picone O, Mandelbrot L, Roques P (2020) Evidence and possible mechanisms of rare maternal-fetal transmission of SARS-CoV-2. J Clin Virol 128:104447. https://doi.org/10. 1016/j.jcv.2020.104447

Fairbrother N, Young AH, Zhang A, Janssen P, Antony MM (2017) The prevalence and incidence of perinatal anxiety disorders among women experiencing a medically complicated pregnancy. Arch Womens Ment Health 20:311-319

Farren J, Jalmbrant M, Ameye L, Joash K, Mitchell-Jones N, Tapp S, Timmerman D, Bourne T (2016) Post-traumatic stress, anxiety and depression following miscarriage or ectopic pregnancy: a prospective cohort study. BMJ Open 6(11):e011864

Favre G, Pomar L, Qi X, Nielsen-Saines K, Musso D, Baud D (2020) Guidelines for pregnant women with suspected SARS-CoV-2 infection. Lancet Infectious

Ferrazzi E, Frigerio L, Savasi V, Vergani P, Prefumo F, Barresi S, Bianchi S, Ciriello E, Facchinetti F, Gervasi MT, Iurlaro E, Kustermann A, Mangili G, Mosca F, Patanè L, Spazzini D, Spinillo A, Trojano G, Vignali M, Villa A, Zuccotti GV, Parazzini 
F, Cetin I (2020) Vaginal delivery in SARS-CoV-2 infected women in northern Italy: a retrospective analysis. BJOG. 127:1116-1121. https://doi.org/10.1111/1471-0528.16278

Finer LB, Zolna MR (2016) Declines in unintended pregnancy in the United States, 2008-2011. N Engl J Med 374(9):843-852

Hao F, Tan W, Jiang L et al (2020) Do psychiatric patients experience more psychiatric symptoms during COVID-19 pandemic and lockdown? A case-control study with service and research implications for immunopsychiatry. Brain Behav Immun 87:100-106. https:// doi.org/10.1016/j.bbi.2020.04.069

Hermann A, Deligiannidis KM, Bergink V et al (2020) Response to SARS-Covid-19-related visitor restrictions on labor and delivery wards in New York City. Arch Womens Ment Health 1-2. https:// doi.org/10.1007/s00737-020-01030-2 [published online ahead of print]

Hoffman SJ, Silverberg SL (2018) Delays in global disease outbreak responses: lessons from H1N1, Ebola, and Zika. Am J Public Health 108(3):329-333. https://doi.org/10.2105/AJPH.2017. 304245

Juan J, Gil MM, Rong Z, Zhang Y, Yang H, Poon LC (2020 May 19) Effects of coronavirus disease 2019 (COVID-19) on maternal, perinatal and neonatal outcomes: a systematic review. Ultrasound Obstet Gynecol 56:15-27. https://doi.org/10.1002/uog.22088

Kimberlin DW, Stagno S (2020) Can SARS-CoV-2 Infection be acquired in utero?: More definitive evidence is needed. JAMA 323(18): 1788-1789. https://doi.org/10.1001/jama.2020.4868

Koc AE et al (2020 Apr) Investigating fear of childbirth in pregnant women and its relationship between anxiety sensitivity and somatosensory amplification. J Obstet Gynaecol 21:1-7

Lackey KA, Pace RM, Williams JE, Bode L, Donovan SM, Järvinen KM, Seppo AE, Raiten DJ, Meehan CL, McGuire MA, McGuire MK (2020 May) SARS-CoV-2 and human milk: what is the evidence? Matern Child Nutr 30:e13032. https://doi.org/10.1111/mcn.13032

Lebel C, MacKinnon A, Bagshawe M, Tomfohr-Madsen L, Giesbrecht G (2020) Elevated depression and anxiety symptoms among pregnant individuals during the COVID-19 pandemic J Affect Disord 277:513. https://doi.org/10.1016/j.jad.2020.07.126

Li N, Han L, Peng M, Lv Y, Ouyang Y, Liu K, Yue L, Li Q, Sun G, Chen L, Yang L (2020) Maternal and neonatal outcomes of pregnant women with COVID-19 pneumonia: a case-control study. Clin Infect Dis:ciaa352. https://doi.org/10.1093/cid/ciaa352

Liu H, Wang LL, Zhao SJ, Kwak-Kim J, Mor G, Liao AH (2020a) Why are pregnant women susceptible to COVID-19? An immunological viewpoint. J Reprod Immunol 139:103122. https://doi.org/10.1016/ j.jri.2020.103122 Epub 2020 Mar 19

Liu N, Zhang F, Wei C, Jia Y, Shang Z, Sun L, Wu L, Sun Z, Zhou Y, Wang Y, Liu W (2020b) Prevalence and predictors of PTSS during COVID-19 outbreak in China hardest-hit areas: gender differences matter. Psychiatry Res 287:112921

Micelli E, Cito G, Cocci A, Polloni G, Russo GI, Minervini A, Carini M, Natali A, Coccia ME (2020) Desire for parenthood at the time of COVID-19 pandemic: an insight into the Italian situation. J Psychosom Obstet Gynaecol 41(3):183-190. https://doi.org/10. 1080/0167482X.2020.1759545

Milgrom J, Hirshler Y, Reece J et al (2019) Social support - a protective factor for depressed perinatal women? Int J Environ Res Public Health 16(8):1429

Minello A (2020) The pandemic and the female academic Nature. https:// doi.org/10.1038/d41586-020-01135-9

Mullins E, Evans D, Viner RM, O'Brien P, Morris E (2020 May) Coronavirus in pregnancy and delivery: rapid review. Ultrasound Obstet Gynecol 55(5):586-592. https://doi.org/10.1002/uog.22014

Nanda K, Lopez LM, Grimes DA et al (2012) Expectant care versus surgical treatment for miscarriage. Cochrane Database Syst Rev 2012(3):CD003518. https://doi.org/10.1002/14651858.CD003518. pub3
National Public Radio. Global lockdowns resulting in 'horrifying surge' in domestic violence, U.N. warns. https:/www.npr.org/sections/ coronavirus-live-updates/2020/04/06/827908402/globallockdowns-resulting-in-horrifying-surge-in-domestic-violence-u-nwarns. Accessed 6 Apr 2020

Organisation for Economic Co-operation and Development (2020). Addressing femicide in the context of rampant violence against women in Latin America. https://www.oecd.org/gender/data/ addressing-femicide-in-the-context-of-rampant-violence-againstwomen-in-latin-america.htm. Accessed Apr 2020

O'Rourke K, Murphy T, Srinivas SK, Pegues DA (2018) Preparing for emerging infectious diseases in the perinatal population. J Obstet Gynecol Neonatal Nurs 47(2):245-253. https://doi.org/10.1016/j. jogn.2017.06.005

Pao C, Guintivano J, Santos H, Meltzer-Brody S (2019) Postpartum depression and social support in a racially and ethnically diverse population of women. Arch Womens Ment Health 22(1):105-114

Ralph LJ, Foster DG, Rocca CH (2020) Comparing prospective and retrospective reports of pregnancy intention in a longitudinal cohort of U.S. women. Perspect Sex Reprod Health 52(1):39-48. https:// doi.org/10.1363/psrh.12134 Epub 2020 Mar 18

Rasmussen SA, Smulian JC, Lednicky JA, Wen TS, Jamieson DJ (2020) Coronavirus disease 2019 (COVID-19) and pregnancy: what obstetricians need to know. Am J Obstet Gynecol 222(5):415-426. https://doi.org/10.1016/j.ajog.2020.02.017

Schwartz RM, Tuminello S, Kerath SM, Rios J, Lieberman-Cribbin W, Taioli E (2018) Preliminary assessment of hurricane harvey exposures and mental health impact. Int J Environ Res Public Health 15(5):974. Published 2018 May 13. https://doi.org/10.3390/ ijerph15050974

Stewart DE, Chandra PS (2017) WPA international competency-based curriculum for mental health providers on intimate partner violence and sexual violence against women. World Psychiatry 16(2):223224. https://doi.org/10.1002/wps.20432

Street AE, Dardis CM (2018) Using a social construction of gender lens to understand gender differences in posttraumatic stress disorder. Clin Psychol Rev 66:97-105

UNICEF (2020) Coronavirus (COVID-19) parenting tips "Expert tips to help you deal with COVID-19 parenting challenges". https://www. unicef.org/coronavirus/covid-19-parenting-tips. Accessed 2 Nov 2020

United Nations (2020) Make the prevention and redress of violence against women a key part of national response plans for COVID19. https://www.un.org/en/un-coronavirus-communications-team/ make-prevention-and-redress-violence-against-women-key-part. Accessed 23 Nov 2018

Wang C, Pan R, Wan X, Tan Y, Xu L, Ho CS, Ho RC (2020a) Immediate psychological responses and associated factors during the initial stage of the 2019 coronavirus disease (COVID-19) epidemic among the general population in China. Int J Environ Res Public Health 17(5):1729

Wang G, Zhang Y, Zhao J, Zhang J, Jiang F (2020b) Mitigate the effects of home confinement on children during the COVID-19 outbreak. Lancet 395(10228):945-947. https://doi.org/10.1016/S01406736(20)30547-X

World Health Organization. Violence against women https://www.who. int/news-room/feature-stories/detail/violence-against-women. Accessed 23 Nov 2018

Wu Y, Zhang C, Liu H, Duan C, Li C, Fan J, Li H, Chen L, Xu H, Li X, Guo Y, Wang Y, Li X, Li J, Zhang T, You Y, Li H, Yang S, Tao X, Xu Y, Lao H, Wen M, Zhou Y, Wang J, Chen Y, Meng D, Zhai J, Ye Y, Zhong Q, Yang X, Zhang D, Zhang J, Wu X, Chen W, Dennis CL, Huang HF (2020) Perinatal depressive and anxiety symptoms of pregnant women along with COVID-19 outbreak in China. Am J Obstet Gynecol 223:240.e1-240.e9. https://doi.org/10. 1016/j.ajog.2020.05.009 
Yan J, Guo J, Fan C et al (2020) Coronavirus disease 2019 in pregnant women: a report based on 116 cases. Am J Obstet Gynecol 223(1): 111.e1-111.e14. https://doi.org/10.1016/j.ajog.2020.04.014

Zaigham M, Andersson O (2020 Apr 7) Maternal and perinatal outcomes with COVID-19: a systematic review of 108 pregnancies. Acta Obstet Gynecol Scand 99:823-829. https://doi.org/10.1111/aogs. 13867
Zeng H, Xu C, Fan J, Tang Y, Deng Q, Zhang W, Long X (2020) Antibodies in infants born to mothers with COVID-19 pneumonia [published online ahead of print, 2020 Mar 26]. JAMA. 323(18): 1848-1849. https://doi.org/10.1001/jama.2020.4861

Publisher's note Springer Nature remains neutral with regard to jurisdictional claims in published maps and institutional affiliations. 